\section{Supra-Annular Transcatheter Aortic Valve Position May Correct Annular Under- Expansion Due to Valvular Asymmetry}

Ozan Mehmet Demir, MD; Satoru Mitomo, MD; Francesco Giannini, MD; Antonio Mangieri, MD; Marco B. Ancona, MD; Matteo Montorfano, MD; Azeem Latib, MD; Antonio Colombo, MD

A 64-year-old woman with previous coronary artery bypass surgery was referred for transcatheter aortic valve implantation (TAVI) for severe aortic stenosis. Echocardiography indicated an aortic valve area of $0.7 \mathrm{~cm}^{2}$ and a mean gradient of $42 \mathrm{mmHg}$. Multi-detector computed tomography (MDCT) confirmed type-1 functional bicuspid aortic valve with fused right and left coronary cusps, and an aortic valve perimeter of $84.5 \mathrm{~mm}$ and area of $561 \mathrm{~mm}^{2}$. The patient underwent transfemoral TAVI using a selfexpanding 27-mm Acurate neo Symetis (Boston Scientific, Massachusetts, US) following pre-dilatation with $20-\mathrm{mm}$ balloon. The TAVI prosthesis appeared well-expanded in the left anterior oblique projection (Figure A) but significant under-expansion was noted in the right anterior oblique projection (Figure B). Despite the absence of aortic regurgitation and a residual mean gradient of $11 \mathrm{mmHg}$ on echocardiography, in view of the markedly asymmetric TAVI prosthesis expansion and unclear long-term clinical correlation, the patient underwent post-dilatation with $20-\mathrm{mm}$ balloon, with good expansion of the balloon (Figure C); nevertheless the prosthesis did not improve its symmetry (Figure D; Supplementary Movie). Final echocardiogram indicated a mean aortic gradient of $11 \mathrm{mmHg}$ and no paravalvular leak. Subsequent MDCT showed poor expansion of the TAVI prosthesis on short-axis view (Figure E,F). Due to the supra-annular position of the prosthetic valve, however, there was round and symmetrical geometry at the level of prosthetic leaflet coaptation (Figure G). The effective office area was $2.8 \mathrm{~cm}^{2}$, suggesting no patient-prosthesis mismatch, but the prosthetic leaflets had a "pin-wheeling" appearance. The radial strength of the TAVI prosthesis may be an important factor with regard to long-term outcome in patients with asymmetric annuli, bicuspid aortic valve and, in particular, for patients with suboptimal postTAVI aortic valve gradients. Of note, lower ratio of postTAVI annular area/pre-TAVI annular area and larger

Received June 14, 2018; revised manuscript received July 12, 2018; accepted July 28, 2018; J-STAGE Advance Publication released online September 1, 2018 Time for primary review: 19 days

Unit of Cardiovascular Interventions, IRCCS San Raffaele Scientific Institute, Milan (O.M.D., S.M., F.G., A.M., M.B.A., M.M., A.L., A.C.); Vita-Salute San Raffaele University, Milan (O.M.D., S.M., F.G., A.M., M.B.A., M.M., A.L., A.C.), Italy; and Department of Cardiology, Hammersmith Hospital, Imperial College Healthcare NHS Trust, London (O.M.D.), UK

Current address: Villa Maria Cecilia Hospital, GVM, Cotignola, RA (A.C.), Italy

Mailing address: Antonio Colombo, MD, IRCCS San Raffaele Scientific Institute, Via Olgettina 60, 20132 Milan, Italy. E-mail: info@emocolumbus.it

ISSN-1346-9843 All rights are reserved to the Japanese Circulation Society. For permissions, please e-mail: cj@j-circ.or.jp

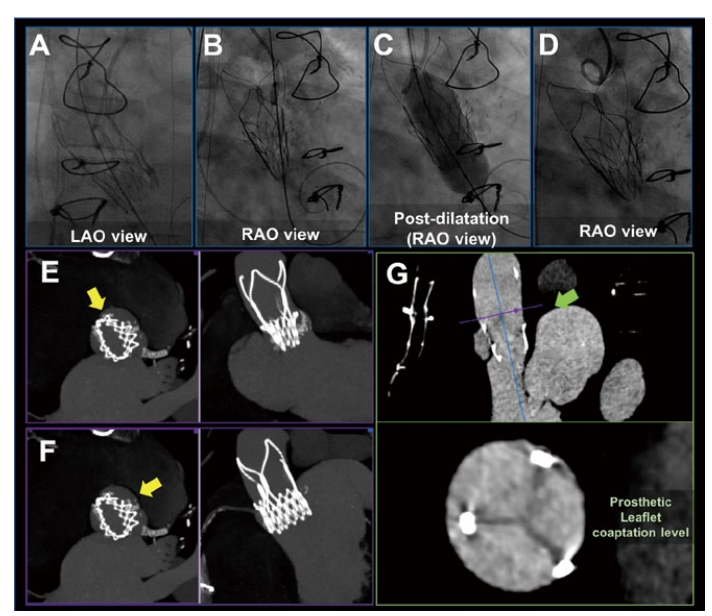

Figure. Peri-procedural fluoroscopy and computed tomography (CT) of self-expanding transcatheter aortic valve implantation (TAVI). (A,B) Fluoroscopy of the TAVI prosthesis (A) well-expanded in left anterior oblique (LAO) projection and (B) under-expanded in right anterior oblique (RAO) projection. (C) Fluoroscopy of post-dilatation with 20-mm balloon with good expansion of the balloon in RAO projection. (D) Fluoroscopy of TAVI prosthesis in RAO projection showing that the asymmetry did not improve following post-dilatation. (E-G) Multi-detector CT showing (E) poor expansion of the TAVI prosthesis on short-axis view (arrow); (F) good expansion of the TAVI prosthesis on long-axis view (arrow); and (G) that due to the supra-annular position of the prosthetic valve there was round and symmetrical geometry at the level of prosthetic leaflet coaptation (arrow)

body surface area have been shown to be independent determinants of severe patient-prosthesis mismatch early after TAVI, indicating the importance of the degree of transcatheter heart valve expansion. ${ }^{1}$ This case shows that despite marked annular asymmetry on fluoroscopy and CT, the supra-annular position of the valve partially corrects this irregularity in an apparent favorable fashion. The impact on long-term durability, however, is unknown.

\section{Acknowledgments / Sources of Funding}

None.

\section{Disclosures}

A.L. has served on the advisory board for Medtronic; and has received honoraria from Abbott Vascular. The other authors declare no conflicts of interest.

\section{Reference}

1. Utsunomiya H, Mihara H, Itabashi Y, Kobayashi S, Siegel RJ, Chakravarty T, et al. Geometric changes in ventriculoaortic complex after transcatheter aortic valve replacement and its association with post-procedural prosthesis-patient mismatch: An intraprocedural 3D-TEE study. Eur Heart $J$ Cardiovasc Imaging 2017; 18: 1-10.

\section{Supplementary Files}

Supplementary Movie. Fluoroscopic acquisition of deployed transcatheter aortic valve implantation prosthesis with projection moving from right to left anterior oblique, demonstrating asymmetric expansion.

Please find supplementary file(s);

http://dx.doi.org/10.1253/circj.CJ-18-0691 